\title{
A dinâmica demográfica e a sustentabilidade do regime geral de previdência social brasileiro
}

\author{
Diana Vaz de Lima \\ Universidade de Brasilia \\ José Matias-Pereira \\ Universidade de Brasília
}

\begin{abstract}
Este trabalho tem como objetivo estudar os efeitos da dinâmica demográfica na sustentabilidade do Regime Geral de Previdência Social (RGPS), a partir das mudanças observadas na estrutura etária da população brasileira e na estrutura etária dos contribuintes e beneficiários do RGPS. Sobre os fatores relacionados com a dinâmica demográfica, os resultados mostram a tendência de queda do número de pessoas mais jovens e do aumento do número de pessoas mais velhas, evidenciando modificação da razão entre as faixas etárias. Com relação às contas previdenciárias, verificou-se que, em 2011, havia menos de $5 \%$ de possibilidade de o resultado previdenciário apresentar-se equilibrado, e que no período de 2012 a 2030 há evidências de que a dinâmica demográfica aumentará em até cinco vezes a necessidade de financiamento observada em 2003.
\end{abstract}

Palavras-chave: Brasil; dinâmica demográfica; Regime Geral de Previdência Social; sustentabilidade.

Dinámica demográfica y la sostenibilidad de la Política General de Bienestar Social brasileño Este trabajo tiene como objetivo estudiar los efectos de la dinámica demográfica en la sostenibilidad de la Política General de Bienestar Social de Brasil (Regime Geral da Previdência Social — RGPS), teniendo en cuenta los cambios observados en la estructura por edades de la población brasileña y en la estructura de edad de los contribuyentes y beneficiarios del RGPS. En cuanto a los factores relacionados con la dinámica demográfica, los resultados muestran una tendencia a la caída en el número de personas más jóvenes y un aumento en el número de personas de edad avanzada, evidenciando el cambio en la relación entre los grupos de edad. En cuanto a las cuentas de la Seguridad Social, en 2011 había menos de $5 \%$ de probabilidad de los gastos de los ingresos de la seguridad social y de seguridad social que son equilibrados, y en el período 2012-2030 existe evidencia de que la dinámica demográfica se incrementarán en hasta cinco veces la necesidad de financiación observado en 2003.

Palabras clave: Brasil; dinámica demográfica; Política General de Bienestar Social; sostenibilidad.

DOI: http://dx.doi.org/10.1590/0034-76121558

Artigo recebido em 30 abr. 2013 e aceito em 25 abr. 2014. 


\begin{abstract}
Demographic dynamics and the sustainability of Brazilian Social Security General Policy This paper aims at studying the effects of demographic dynamics in the sustainability of Brazilian Social Security General Policy (Regime Geral da Previdência Social - RGPS), taking into account the changes observed in the age structure of the Brazilian population and of taxpayers and beneficiaries of RGPS. About the factors related with demographic dynamics, the results show a falling tendency in the number of younger people and an increase in the number of older people, highlighting the change in the ratio between age groups. Concerning social security accounts, in 2011 there was less than $5 \%$ of chance of the social security result be balanced, and in the period 2012-2030 there are evidences that the demographic dynamics will increase up to five times the need for financing observed in 2003.
\end{abstract}

Keywords: Brazil; demographic dynamics; Social Security General Policy; sustainability.

\title{
1. Introdução
}

Os efeitos da dinâmica demográfica e a sustentabilidade dos regimes previdenciários de responsabilidade dos governos nacionais têm gerado preocupações em todo o mundo (Croix, Pierrard e Sneessens, 2013; Bloom et al., 2007). De acordo com Queiroz e Fígoli (2011), o Brasil está enfrentando problema semelhante ao de nações desenvolvidas, que com o envelhecimento da população estão procurando maneiras de aumentar a idade média de aposentadoria e minimizar o impacto sobre o setor público.

A esse respeito, o Banco Mundial destaca nas conclusões do relatório Becoming old in an older Brazil: implications of population aging on growth, poverty, public finance and service delivery que o Brasil está em meio a uma profunda transformação socioeconômica guiada pela mudança demográfica, que apresenta, dentre outros, uma acentuada queda da taxa de fecundidade e o aumento da expectativa de vida (World Bank, 2011), modificando, na visão de Kieling (2009), o peso proporcional dos diversos grupos de idade no conjunto da população.

Um dos efeitos da mudança do peso proporcional dos grupos de idade é a relação da razão de dependência total, que representa a proporção dos jovens e idosos em relação à população economicamente ativa e expressa a transferência entre gerações. De acordo com Brito (2008), enquanto no ano 2000 no Brasil a razão de dependência dos jovens era quase seis vezes maior do que a dos idosos, em 2050 as duas razões de dependência serão praticamente iguais.

Em busca de mecanismos que permitam a sustentabilidade das contas previdenciárias, discute-se no âmbito do governo brasileiro a implantação de uma idade mínima progressiva e o aumento do tempo de contribuição. Contudo, apesar de as estatísticas populacionais e dos indicadores demográficos sinalizarem a urgência do tema, ainda não há um amplo debate sobre os efeitos que a dinâmica demográfica traz para a sustentabilidade do regime geral de previdência social brasileiro.

A partir desse cenário, considerando que a previdência social brasileira é de caráter contributivo e de filiação obrigatória, observados critérios que preservem o equilíbrio financeiro e atuarial (art. 201 da Constituição de 1988), e que cabe ao Estado garantir ao indivíduo que 
não tenha mais capacidade laborativa os recursos necessários à sua sobrevivência e a de seus dependentes na proporção dos benefícios definidos pela legislação, tem-se a seguinte questão de pesquisa: quais efeitos a dinâmica demográfica traz para a sustentabilidade do regime geral de previdência social brasileiro?

Nesse sentido, o objetivo geral desta pesquisa consiste em estudar os efeitos da dinâmica demográfica na sustentabilidade do regime geral de previdência social brasileiro. Mais especificamente, este trabalho destina-se a mensurar os efeitos da dinâmica demográfica nas receitas previdenciárias e nas despesas previdenciárias do RGPS brasileiro para o período de 2003 a 2030, e as perspectivas de equilíbrio das contas previdenciárias a partir dos resultados apurados.

Para tanto, foram realizados levantamentos, estimativas e projeções da situação financeira do RGPS para o período de 2003 a 2030 a partir de um conjunto de premissas demográficas e econômicas (aggregate accounting), com o uso de modelos analíticos e da técnica de simulação de Monte Carlo (MC), consubstanciados nas estimativas e projeções elaboradas pelo Instituto Brasileiro de Geografia e Estatística (IBGE) e na base de dados Aeps Infologo.

Para atender a questão da pesquisa, o artigo apresenta, além dessa introdução: (i) os aspectos conceituais da transição demográfica e sua repercussão em previdência; (ii) os fatores componentes da dinâmica demográfica brasileira; (iii) procedimentos metodológicos; (iv) análises e resultados do estudo; (v) considerações finais.

\section{Transição demográfica e previdência}

Tendo como base as pesquisas de Nam (1994) e Ryder (1984), Blue e Espenshade (2011) esclarecem que o conceito de transição demográfica geralmente é atribuído a Adolphe Landry e Warrem Thompson, que no século XX foram os primeiros a descrever um padrão regular de mudança da população, e mais tarde a Kingsley Davis e Frank Notestein, que correlacionaram as mudanças na estrutura populacional com as tendências de desenvolvimento econômico e social.

Para os autores, embora os pesquisadores ainda não tenham chegado a um acordo sobre uma teoria que explique as causas da transição demográfica, a investigação empírica documentou padrões comuns de experiência demográfica entre as populações. Partindo do entendimento de que essas populações podem diferir no tempo de transição, no nível de desenvolvimento econômico e social no qual ocorre a transição e na interferência das questões religiosas sobre as decisões a respeito da fertilidade, consideram a transição demográfica uma descrição generalizada de tendências e não um caminho fixo que todas as populações devem seguir (Blue e Espenshade, 2011).

Para Brito (2008), a transição demográfica nos países em desenvolvimento, latino-americanos e asiáticos, tem sido mais acelerada do que nos países desenvolvidos. Contudo, Blue e Espenshade (2011) esclarecem que, apesar de países em desenvolvimento terem crescido a uma taxa média anual de mais de $2 \%$ após a Segunda Guerra Mundial, alguns com ganhos 
populacionais anuais de até 4\%, houve rápido declínio da fecundidade em apenas algumas décadas. Para esses pesquisadores, embora grande parte da África subsaariana ainda tenha fecundidade bem acima do nível de reposição, a maioria do resto do mundo parece ter completado a transição demográfica.

A partir desse cenário, Blue e Espenshade (2011) entendem que o conceito de transição demográfica descreve tanto a experiência do mundo em desenvolvimento como a experiência do mundo desenvolvido, destacando que entre as principais diferenças entre esses dois mundos estão a velocidade de declínio da mortalidade e da fecundidade e a taxa de crescimento da população.

Paiva e Wajnman (2005) trazem a crença de que em países em desenvolvimento, em particular na América Latina, haveria a possibilidade de se tirar proveito da redução da taxa de dependência, uma vez que esses países passam pela fase em que o crescimento populacional tem efeito positivo sobre o crescimento econômico (bônus demográfico).

Para Pessoa (2009), essa processualidade da transição demográfica traduz o processo de crescimento populacional, afetando, consequentemente, a composição etária das populações, uma vez que as mudanças no ritmo de crescimento e de decrescimento das taxas de natalidade e de mortalidade modificam a razão entre as faixas etárias.

Em questões de previdência, Matias-Pereira (2010) considera que, a exemplo do Brasil, a transição demográfica se apresenta como uma ameaça concreta para a grande maioria dos países do mundo. Em outro estudo traz que:

As projeções indicam que os países da Europa (UE), em 2030, contarão com 20 milhões de pessoas a menos na sua força de trabalho. Os cálculos atuariais mostram que o sistema previdenciário, mantida essa tendência, não poderá suportar os gastos decorrentes dessas mudanças demográficas. Para as autoridades da União Europeia não existe alternativa: trabalhar até mais tarde, ou redução no valor das aposentadorias. Será preciso, ainda, gerar estímulos para aumentar a natalidade. (Matias-Pereira, 2008:70)

Ao analisar o futuro da previdência social, Gonzalez-Eiras e Niepel (2008) entendem que as transferências intergeracionais surgem na ausência de compromissos ou estratégias. Para os pesquisadores, soluções fechadas para fazer frente à previsão do envelhecimento da população a partir da elevação das taxas de contribuições previdenciárias trazem baixos benefícios para os aposentados, uma vez que respostas para choques demográficos por meio de alíquotas de equilíbrio reduzem o consumo na velhice.

No caso do Brasil, Paiva e Wajnman (2005) esclarecem que o impacto da transição demográfica sobre a previdência social é uma questão bastante complexa, porque o sistema brasileiro já opera em desequilíbrio estrutural.

A preocupação com os efeitos das transformações demográficas na previdência social brasileira também está registrada em pareceres oficiais emitidos por órgãos do governo. Em fiscalização realizada no Ministério da Previdência Social (MPS) no período de janeiro de 2007 a dezembro de 2009, o Tribunal de Contas da União (TCU) alertou sobre a gravidade 
da situação, recomendando a realização de estudos com o propósito de alterar dispositivos legais para, entre outros, possibilitar mudanças paramétricas no RGPS a longo prazo, visando minimizar os efeitos da pressão demográfica sobre o regime (TCU, 2010).

No relatório do anexo de metas fiscais relativo às projeções atuariais para o RGPS em 2012 (MPS, 2012a), o MPS trouxe que, pelo fato de o RGPS cobrir potencialmente qualquer indivíduo da população brasileira que não esteja filiado ao regime próprio de previdência social (RPPS), destinado aos servidores públicos, a dinâmica demográfica é uma das principais variáveis a determinar a evolução da razão entre contribuintes e beneficiários. De acordo com o relatório, "a taxa de crescimento da população, a evolução de seu perfil etário e a taxa de urbanização são variáveis fundamentais para estimar a evolução dos contribuintes e beneficiários" (MPS, 2012a:13).

Para Matos e Pinto (2012), o forte processo de envelhecimento, o aumento progressivo da longevidade e as baixas taxas de natalidade estão entre os fatores que ocasionam a diminuição da base de financiamento e o aumento das despesas com benefícios no Sistema brasileiro: enquanto na década de 1940 registravam-se mais de 30 contribuintes por beneficiário, atualmente essa proporção está menos de três para um.

\section{Fatores componentes da dinâmica demográfica brasileira}

\subsection{Estimativas e projeções do IBGE}

Estimativas e projeções do IBGE mostram que o efeito combinado da redução dos níveis de fecundidade e da mortalidade no Brasil tem produzido transformações no padrão etário da população brasileira. Avaliando a participação relativa da população brasileira por grupos de idade na população total nos anos de 1980, 2010 e 2030, evidencia-se a modificação da razão entre as faixas etárias mais jovens e mais velhas (gráfico 1).

Gráfico 1

Participação relativa da população brasileira por grupos de idade

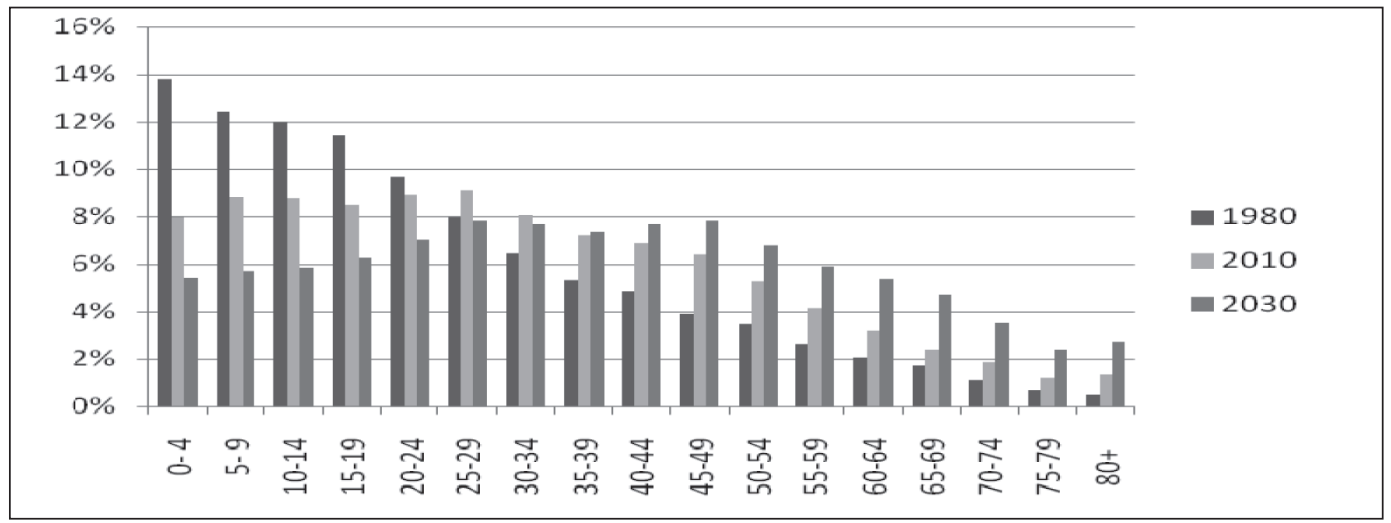

Fonte: IBGE (2008).

Rev. Adm. Pública - Rio de Janeiro 48(4):847-868, jul./ago. 2014 
No caso dos idosos brasileiros, potenciais aposentados do RGPS, a razão de dependência $^{1}$ apresenta trajetória crescente em todo o período, tanto de acordo com a metodologia do IBGE (inatividade acima de 65 anos) quanto de acordo com a metodologia do RGPS (inatividade acima dos 60 anos), ou seja, há cada vez mais idosos comparativamente à população economicamente ativa (gráfico 2).

Gráfico 2

Evolução da razão de dependência dos idosos 1980-2030

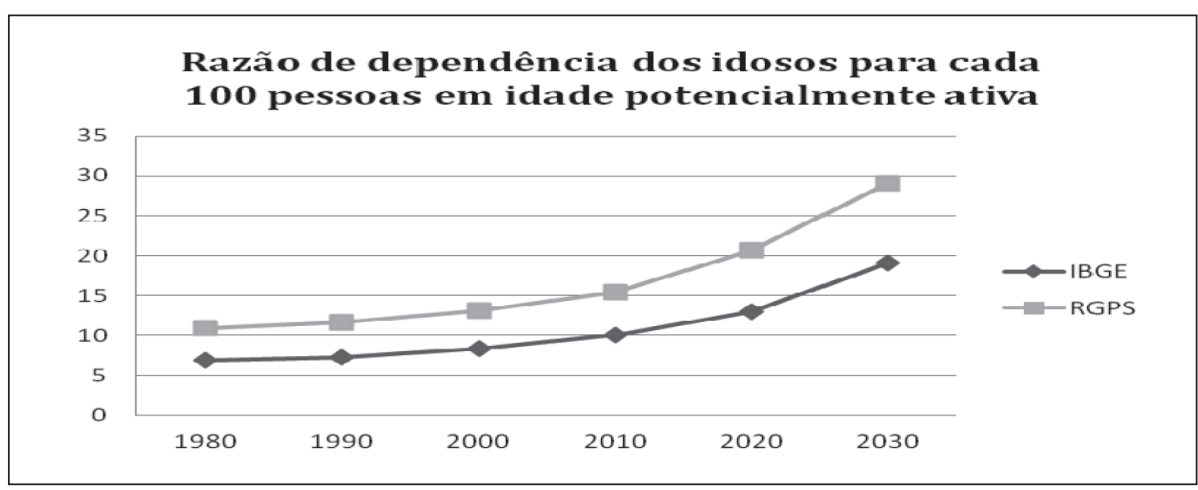

Fonte: IBGE (2008) nos grupos de idade selecionados.

Batista e colaboradores (2009) ratificam esse entendimento, trazendo que as projeções realizadas para a razão de dependência e o índice de envelhecimento apontam para uma importante reorganização no quadro demográfico nacional, com impactos relevantes na sociedade brasileira e nas políticas públicas.

\subsection{Contribuintes pessoas físicas do RGPS - período de 2003 a 2011}

Quando se analisa o número de contribuintes pessoas físicas por grupos de idade no período de 2003 a 2011, a partir da base de dados Aeps Infologo, verifica-se que houve modificação da razão entre as faixas etárias mais jovens e mais velhas, a exemplo das projeções do IBGE. Ao se analisar a evolução do número total de contribuintes do RGPS em cada grupo de idade, verifica-se que a mesma apresentou trajetória crescente em todo o período e em todos os grupos de idade analisados, conforme gráfico 3.

\footnotetext{
${ }^{1}$ Indicador que expressa a proporção de pessoas em idade potencialmente inativa de uma população, em relação a 100 pessoas em idade potencialmente ativa ou disponível para as atividades econômicas.
} 
Evolução de contribuintes do RGPS por grupos de idade 2003-11
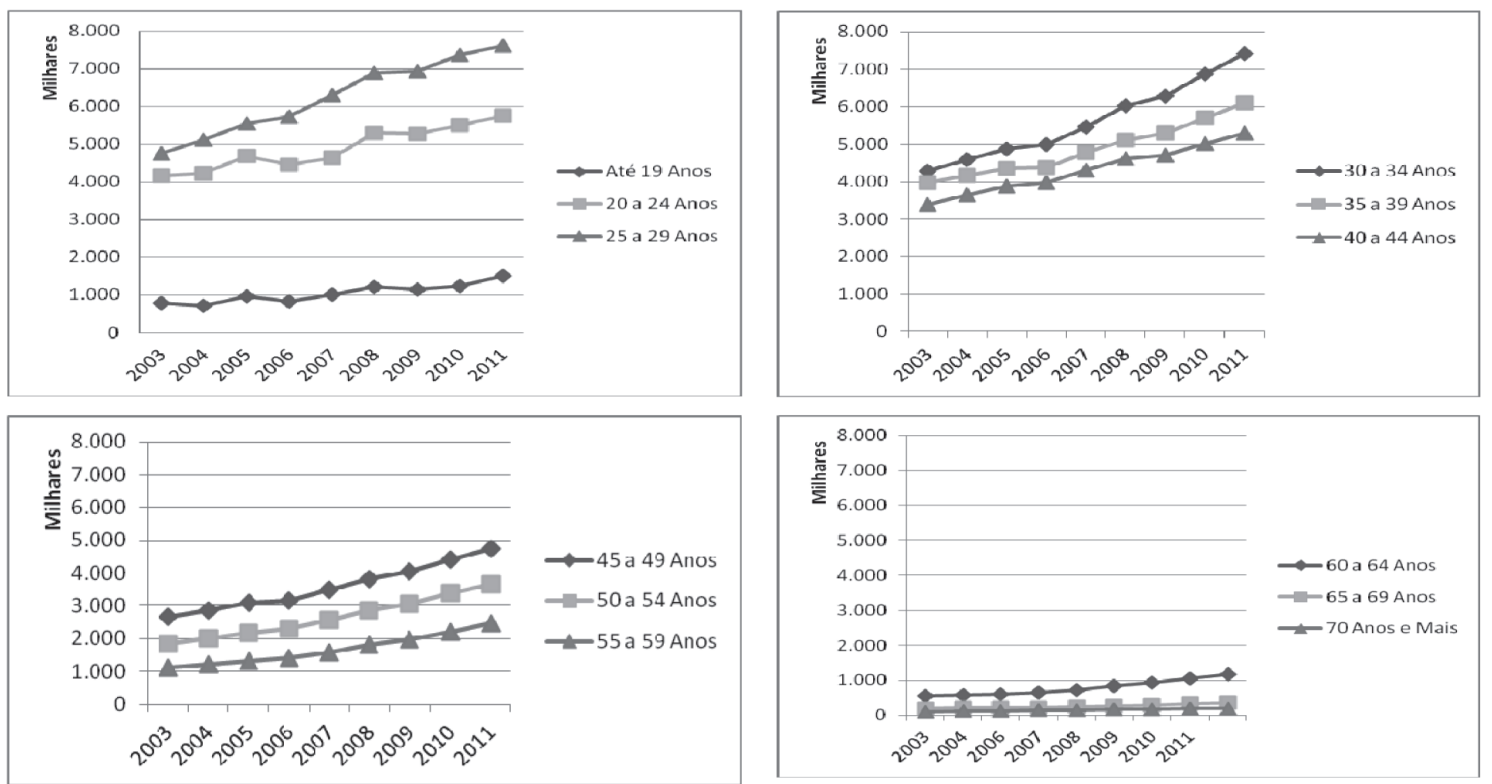

Fonte: Elaboração própria, a partir do número médio mensal de contribuintes pessoas físicas por grupos de idade 2003-21. Base de dados históricos da Previdência Social - Aeps Infologo.

Em termos comparativos, verifica-se que, enquanto o número total de contribuintes do RGPS cresceu quase 70\% no período, o crescimento total da população brasileira acima de 15 anos não chegou a 15\% (dados do IBGE), revelando que houve aumento do número de contribuintes acima do crescimento populacional.

\subsection{Benefícios ativos do RGPS - período de 2003 a 2011}

Ao se analisar a evolução do número de benefícios ativos por grupos de idade no período de 2003 a 2011, verifica-se que houve aumento em torno de 40\% do número total de benefícios ativos em 2011 comparativamente a 2003 (gráfico 4).

A partir dos dados analisados, merece destaque o aumento do número de benefícios ativos para os segurados acima de 50 anos de idade e pensionistas, ratificando que o envelhecimento populacional já vem afetando o perfil dos beneficiários do RGPS. 
Gráfico 4

Evolução do número de benefícios ativos entre 2003 e 2011

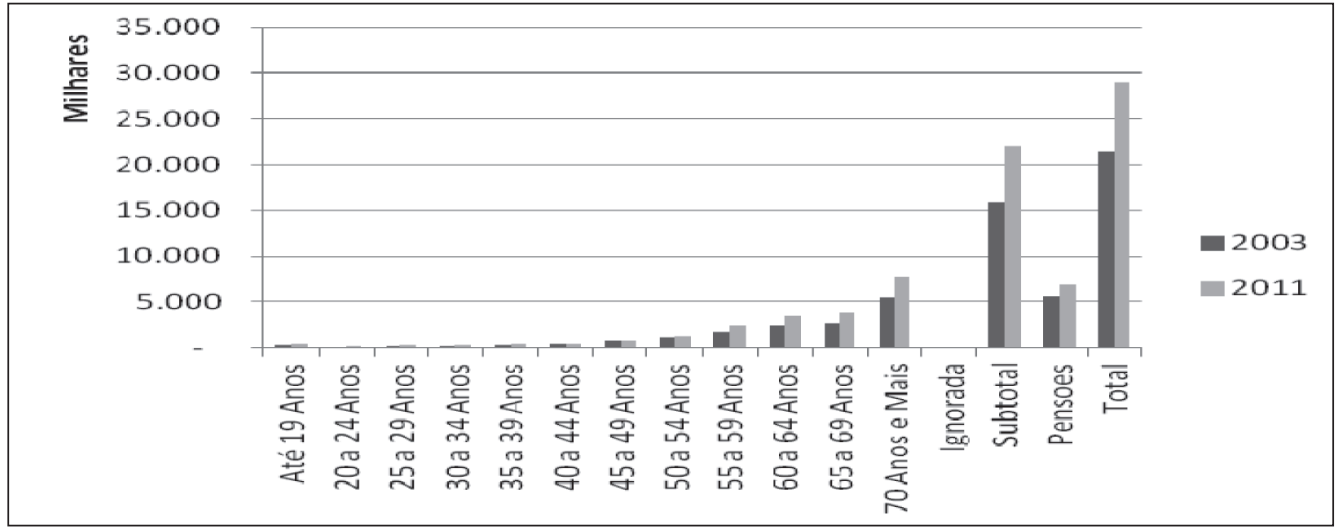

Fonte: IBGE (2008).

\section{Procedimentos metodológicos}

\subsection{Tipo e método de pesquisa}

Considerando que a preocupação principal do estudo é a explicação de um fenômeno, objetivando investigar os efeitos da dinâmica demográfica na sustentabilidade do regime geral de previdência social brasileiro, a pesquisa é do tipo explicativa.

Quanto ao procedimento técnico, para permitir a compreensão dos efeitos da dinâmica demográfica nas contas previdenciárias e prever seu comportamento sob determinadas condições, foram realizados levantamentos, estimativas e projeções da situação financeira do RGPS para o período de 2003 a 2030, a partir de um conjunto de premissas demográficas e econômicas (aggregate accounting), com o uso de modelos analíticos e da técnica de simulação de Monte Carlo (MC), consubstanciados nas estimativas e projeções elaboradas pelo IBGE e na base de dados Aeps Infologo.

\subsection{Seleção e composição da amostra}

Para analisar as transformações no padrão etário da população brasileira, foram extraídos os seguintes dados do estudo Projeção da população do Brasil por sexo e idade - 1980-2050, publicado pelo IBGE: a) número de pessoas por grupos de idade (dados quinquenais), no período de 1980 a 2030; b) número de pessoas de 16 a 59 anos (idade simples), no período de 2003 a 2030; c) número de pessoas acima de 60 anos (idade simples), no período de 2003 a 2030.

Na análise do número de contribuintes, do número de benefícios, das receitas e das despesas do RGPS, os seguintes dados foram extraídos do banco de dados Aeps Infologo — Base de 
dados históricos da previdência social: (i) valor das remunerações de contribuintes pessoas físicas por faixa de idade, no período de 2003 a 2011; (ii) valor dos benefícios ativos (sem pensões) segundo grupos de idade do segurado no período de 2003 a 2011; (iii) valor das pensões ativas segundo grupos de idade do beneficiário no período de 2003 a 2011; (iv) fluxo de caixa consolidado do INSS no período de 2003 a 2011; (v) alíquotas de contribuição vigentes para a previdência social do empregado no período de 2003 a 2011; (vi) número médio mensal de contribuintes pessoas físicas por grupos de idade, no período de 2003 a 2011; (vii) quantidade de benefícios ativos por idade do segurado no período de 2003 a 2011; (viii) quantidade de pensões ativas por idade do beneficiário no período de 2003 a 2011; (ix) número de pessoas de 10 anos ou mais de idade, por condição de atividade (economicamente ativa e não economicamente ativa) na semana de referência e sexo, segundo os grupos de idade, no período de 2003 a 2010; (x) número de pessoas de 16 a 59 anos de idade, ocupadas na semana de referência, por proteção previdenciária e sexo, segundo os grupos de idade, no período de 2003 a 2010.

Para que a metodologia fosse consistente durante todo o período analisado, esses valores foram corrigidos pelo Índice Nacional de Preços ao Consumidor (INPC), base de dezembro de 2011, efetuando o deflacionamento pelo índice médio anual. O INPC mede o custo de vida das famílias com rendimentos entre um e cinco salários mínimos, e é o índice oficialmente utilizado pelo INSS para reajustar os benefícios previdenciários emitidos.

Para que fosse possível relacionar o banco de dados do IBGE com o banco de dados do Aeps Infologo, as projeções foram agregadas em 12 grupos de idade: até 19 anos; de 20 a 24 anos; de 25 a 29 anos; de 30 a 34 anos; de 35 a 39 anos; de 40 a 44 anos; de 45 a 49 anos; de 50 a 54 anos; de 55 a 59 anos; de 60 a 64 anos; de 65 a 69 anos; e de 70 anos e mais.

\subsection{Desenvolvimento das hipóteses e definição dos modelos empregados}

A pesquisa teve como principal hipótese que a dinâmica demográfica afeta a sustentabilidade do regime geral de previdência social brasileiro. Nessa perspectiva, buscou-se construir um modelo com variáveis que permitissem estimar o efeito da dinâmica demográfica nas receitas previdenciárias e nas despesas previdenciárias do RGPS.

Para o período de 2003 a 2011, com relação às premissas econômicas, o estudo considerou as características do mercado de trabalho brasileiro e as projeções elaboradas pelo Ministério da Previdência Social para atender a Lei de Responsabilidade Fiscal (MPS, 2012a), bem como o montante das receitas previdenciárias e das despesas previdenciárias fornecido pela base de dados Aeps Infologo.

Com o intuito de compreender os efeitos futuros da dinâmica demográfica e as perspectivas de equilíbrio no resultado previdenciário, foram utilizados modelos analíticos e aplicada a técnica de simulação de MC, sendo analisados dois cenários: o primeiro considerou que a evolução do número de contribuintes pessoas físicas e de benefícios ativos do RGPS para o período de 2012 a 2030 seria 100\% equivalente à evolução observada em cada grupo de idade da população brasileira projetada pelo IBGE (razão demográfica), apurada a partir do quociente entre a quantidade de pessoas da população brasileira em determinado grupo de 
idade no ano $t$ e a quantidade de pessoas também da população brasileira em determinado grupo de idade no ano $t-1$.

No segundo cenário de projeção para o período de 2012 a 2030, além da "razão demográfica", as receitas foram ajustadas por uma taxa anual de crescimento da produtividade em $1,6 \%$. No caso das despesas, além da "razão demográfica", foi aplicado um reajuste médio de benefícios a partir de estudo do Ministério da Previdência (MPS, 2012a) e considerado crescimento vegetativo do valor da despesa na ordem de 2,27\% (diferença ponderada entre valores de benefícios concedidos e emitidos).

\subsubsection{Período de 2003 a 2011}

No estudo, a modelagem da receita previdenciária para o período de 2003 a 2011 teve como parâmetro a arrecadação líquida (recebimentos próprios menos transferências a terceiros), estimada a partir do número médio mensal de contribuintes pessoas físicas por grupos de idade multiplicado pelo valor médio da contribuição média anual em cada ano e grupo de idade (equação 1):

\section{Equação 1}

$$
\operatorname{Rec} \operatorname{Prev}_{t}{ }_{t}^{a, b}=N \text {. Contrib }{ }_{t}^{a, b} \times \text { VmCont }{ }_{t}^{a, b}
$$

Em que RecPrev $_{t}$ representa a receita previdenciária estimada no ano t; N.Contrib indica o número de contribuintes pessoas físicas no ano t; VmCont refere-se ao valor médio da contribuição no ano t; e $a, b$ tratam da entrada e saída do contribuinte no grupo de idade em análise.

No caso da modelagem da despesa previdenciária para o período de 2003 a 2011, foi construída a partir do número de benefícios ativos em cada ano e grupo de idade e do valor médio dos benefícios pagos em cada período. No caso das pensões, como são tratadas à parte na base de dados Infologo, essas informações foram adicionadas ao final da equação para compor o valor da despesa previdenciária apurada em cada ano (equação 2).

\section{Equação 2}

$$
\text { Desp Prev }_{t}^{a, b}=\left(N \text {. Benef }{ }_{t}^{a, b} \times \text { VmBenef }_{t}^{a, b}\right)+\left(\text { N.Pensões }_{t} \times \text { VmPensões }_{t}\right)
$$

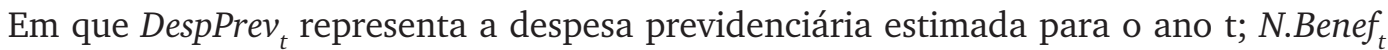
representa a quantidade de benefícios ativos (menos pensões) no ano t; $V m$ Benef $f_{t}$ refere-se ao valor médio anual dos benefícios ativos no ano t; $a, b$ correspondem à entrada e saída do beneficiário do grupo de idade em análise; N.Pensões ${ }_{t}$ refere-se ao número de pensões no ano t; e VmPensões representa o valor médio anual das pensões no ano t. 


\subsubsection{Período de 2012 a 2030}

\section{A. ModelaGem da ReCEITA PREVIDENCIÁRIA}

No primeiro cenário da projeção da receita previdenciária para o período de 2012 a 2030, foi considerado que a evolução do número de contribuintes será 100\% equivalente ao crescimento da população brasileira em cada grupo de idade, mantido o valor médio da contribuição de 2011 (corrigido pelo INPC) apenas ajustado em razão da distribuição dos valores relacionados aos contribuintes ignorados (equação 3).

\section{Equação 3}

$$
\text { Rec Prev }{ }_{t}^{a, b}=\left(\sum N \text {. Contrib }{ }_{t}^{a, b} \times R D_{t}^{a, b}\right) \times \text { VmCont }_{t}^{a, b}
$$

Em que RecPrev $_{t}$ representa a receita previdenciária estimada no ano t; $N$. Contrib ${ }_{t}$ indica o número de contribuintes pessoas físicas no ano $\mathrm{t}$; $R D_{t}$ trata da razão demográfica observada

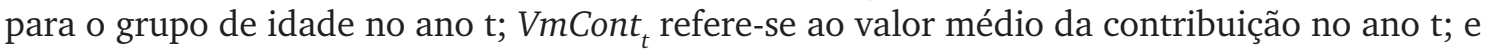
$a, b$ representam a idade de entrada e saída do contribuinte no grupo em análise.

No segundo cenário da projeção da receita previdenciária para o período de 2012 a 2030, além do aumento do número de contribuintes pela equivalência da razão demográfica, o valor da contribuição média anual foi acrescido em 1,6\%, acumulado anualmente, relativo ao crescimento médio da produtividade (equação 4).

\section{Equação 4}

$$
\operatorname{Rec}_{t} \operatorname{Prev}_{t}^{a, b}=\left(\sum N \text {. Contrib }_{t}^{a, b} \times R D_{t}^{a, b}\right) \times\left(\text { VmCont }_{t}^{a, b} \times C P_{t}\right)
$$

Em que RecPrev $_{t}$ representa a receita previdenciária estimada no ano t; $N$. Contrib $t_{t}$ indica o número de contribuintes pessoas físicas no ano $t ; R D_{t}$ trata da razão demográfica observada para o grupo de idade no ano t; VmCont $t_{t}$ refere-se ao valor médio da contribuição no ano t em cada grupo de idade; $a, b$ representam a entrada e saída do contribuinte no grupo de idade em análise; e $C P$ indica o crescimento da produtividade no ano t.

\section{B. MODELAGEM DA DESPESA PREVIDENCIÁRIA}

Na modelagem do primeiro cenário da projeção da despesa previdenciária para o período de 2012 a 2030, foi considerado que a evolução do número de benefícios ativos será 100\% 
equivalente ao crescimento da população brasileira em cada grupo de idade, e que as pensões serão acrescidas para apuração da despesa total com benefícios pagos. Foi mantido o valor do benefício ativo pago em 2011 (corrigido pelo INPC), ajustado apenas pela distribuição dos valores relacionados aos benefícios ativos ignorados (equação 5).

\section{Equação 5}

Desp Prev ${ }_{t}^{a, b}=\left[\left(\sum N\right.\right.$. Benef $\left.{ }_{t}^{a, b} \times R D_{t}^{a, b}\right) \times$ VmBenef $\left._{t}^{a, b}\right]+\left[\left(\sum N\right.\right.$.Pensões $_{t} \times$ CmRD $\left._{t}\right) \times$ VmPensões $\left._{t}\right]$

Em que Desprev ${ }_{t}$ representa a despesa previdenciária estimada para o ano t; N.Benef $f_{t}$ indica a quantidade de benefícios ativos (menos pensões) no ano t; $R D_{t}$ trata da razão demográfica observada para o grupo de idade no ano t; $V m$ Benef ${ }_{t}$ refere-se ao valor médio dos benefícios ativos no ano t; $a, b$ correspondem à entrada e saída do beneficiário do grupo de idade em análise; N.Pensões indica a quantidade de pensões no ano t; $C m R D_{t}$ representa o crescimento médio da razão demográfica no ano t; e VmPensões ${ }_{t}$ refere-se ao valor médio das pensões pago no ano t.

No segundo cenário da projeção da despesa previdenciária para o período de 2012 a 2030, além do aumento do número de benefícios ativos pela equivalência da razão demográfica, o valor do benefício médio anual foi ajustado pelo reajuste real médio projetado para o período (salário mínimo e demais benefícios) e pelo crescimento vegetativo de $2,27 \%$ nos valores dos benefícios pagos. O valor das pensões também foi acrescido para apuração da despesa total com os benefícios pagos (equação 6).

\section{Equação 6}

$$
\begin{aligned}
& \text { Desp Prev }{ }_{t}^{a, b}=\left[\left(\sum N \text {. Benef }{ }_{t}^{a, b} \times R D_{t}^{a, b}\right) \times \mid\left(\text { VmBenef }_{t}^{a, b} \times \text { RBen }_{t} \times \text { CVDesp }_{t}\right) \mid\right]+ \\
& {\left[\left(\sum N . \text { Pensões }_{t} \times \mathrm{CmRD}_{t}\right) \times\left(\text { VmPensões }_{t} \times \text { RBen }_{t} \times \text { CVDesp }_{t}\right)\right]}
\end{aligned}
$$

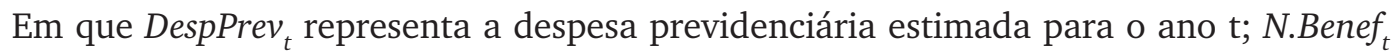
indica a quantidade de benefícios ativos (menos pensões) no ano t; $R D_{t}$ trata da razão demográfica observada para o grupo de idade no ano t; $V m$ Benef ${ }_{t}$ refere-se ao valor médio dos benefícios ativos no ano t; $a, b$ correspondem à entrada e saída do beneficiário do grupo de

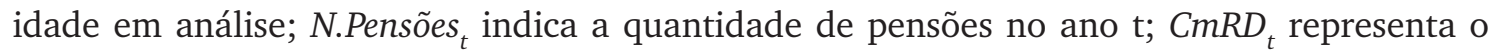
crescimento médio da razão demográfica no ano t; VmPensões ${ }_{t}$ refere-se ao valor médio das pensões pago no ano t; $R B e n_{t}$ indica o reajuste real médio projetado para o ano t; CVDesp $p_{t}$ representa o crescimento vegetativo do valor da despesa no ano t. 


\section{Modelagem do RESULTADO PREVIDENCIÁRIO}

Na apuração do resultado previdenciário, consoante ao modelo de repartição simples, será confrontada a evolução da receita estimada com a evolução da despesa estimada, para todos os grupos de idade e em todos os anos selecionados para o estudo (2003 a 2030), conforme explicitado na equação 7.

Equação 7

Res Prev $_{t}^{a, b}=\frac{\operatorname{RecPrev}_{t}^{a, b}}{\text { DespPrev }_{t}^{a, b}}$

Em que $\operatorname{ResPrev}_{t}$ representa o resultado previdenciário no tempo t; $\operatorname{RecPrev}_{t}$ indica a receita previdenciária no tempo $\mathrm{t}$; DespPrev ${ }_{t}$ refere-se à despesa previdenciária no ano $\mathrm{t}$; e $a, b$ representam a idade de entrada e saída do grupo de idade em análise. Os desequilíbrios eventualmente verificados podem indicar a necessidade de correções no sistema, de forma que a despesa previdenciária seja equivalente à receita previdenciária.

\section{Análise e resultados}

\subsection{Análises e resultados do período de 2003 a 2011}

Efetuando a análise da evolução da receita por grupos de idade no período de 2003 a 2011, observa-se que a trajetória de crescimento da receita se manteve em todo o período, mas houve queda da participação na faixa de 20 a 24 anos e de 35 a 49 anos — justamente quando os indivíduos se encontram no ápice da idade ativa. Nas faixas de até 19 anos e acima de 50 anos observa-se uma maior participação em 2011 na receita previdenciária arrecadada comparativamente a 2003. Outro dado observado diz respeito ao valor real médio da contribuição, que cresceu em torno de $20 \%$ no período analisado.

A partir da análise da evolução da despesa total no período de 2003 a 2011, incluindo os benefícios de pensões, verifica-se trajetória de crescimento da despesa previdenciária em todos os anos observados, mas houve queda da participação da despesa nas faixas de 25 a 59 anos de idade e aumento da participação nos demais grupos de idade, bem como no caso das despesas com pensões. De acordo com os dados, também houve aumento real de quase 40\% nos valores pagos no ano de 2011 comparativamente ao ano de 2003.

Com relação ao resultado previdenciário total no período de 2003 a 2011, os dados mostram que houve déficit em todos os anos analisados, e que os grupos de idade mais velhos foram os que mais influenciaram o resultado previdenciário total, sendo que essa influência aumentou acentuadamente no período observado (gráfico 5). 
Gráfico 5

Participação de grupos de idade: resultado previdenciário 2003-2011

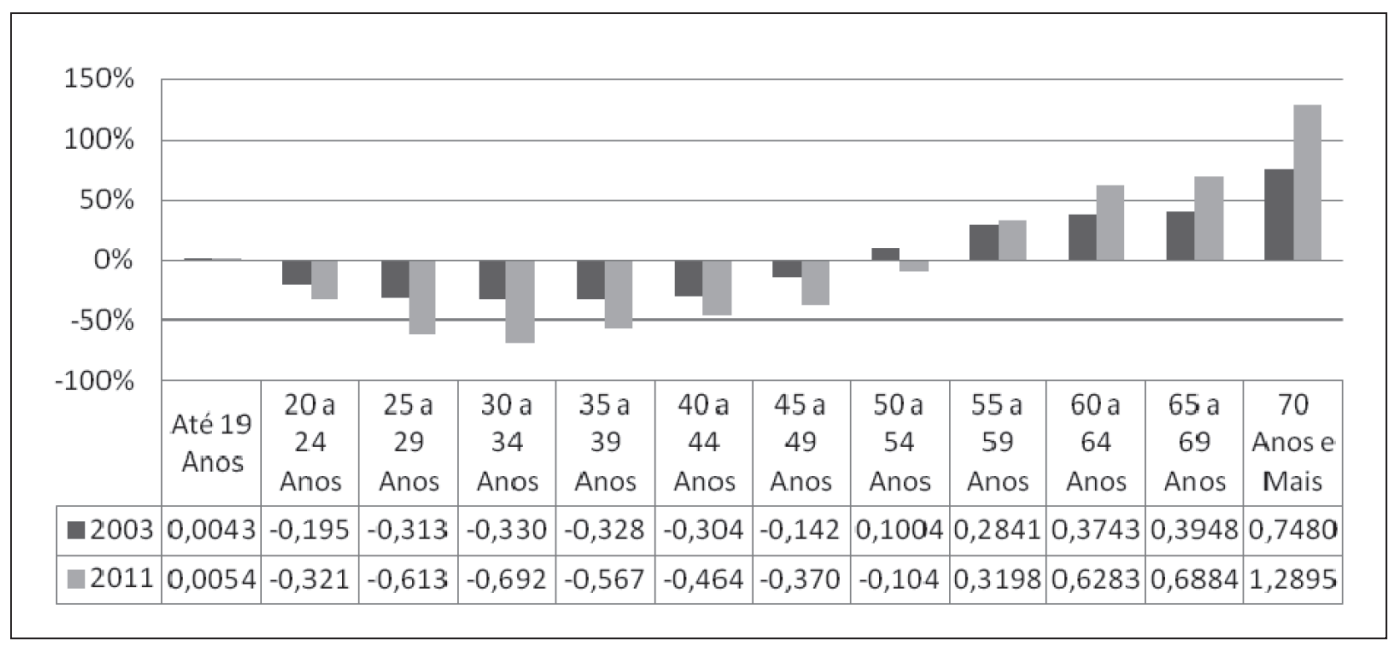

Fonte: Elaboração própria, a partir do Aeps Infologo.

\subsection{Análises e resultados do período de 2012 a 2030}

Quando se compara a participação de cada grupo de idade na evolução da arrecadação da receita no ano de 2030 comparativamente ao ano de 2012, tanto para o Cenário 1 quanto para o Cenário 2, verifica-se que houve redução da capacidade contributiva das coortes mais jovens (até 29 anos). Em ambos os cenários também se verifica que o ritmo de crescimento da receita previdenciária projetado para o período de 2012 a 2030 é menor do que o ritmo de crescimento observado no período de 2003 a 2011, sendo ainda observada a modificação no perfil etário dos contribuintes, sinalizando a prevalência de crescimento da arrecadação nos grupos de idade mais velhos.

Com relação à despesa projetada para o período de 2012 a 2030, no primeiro cenário observa-se crescimento da despesa previdenciária em todos os anos, com tendência de queda do número de benefícios ativos na faixa de até 29 anos e aumento significativo das despesas com benefícios ativos a partir de 65 anos de idade. A análise do segundo cenário da projeção da despesa para o período de 2012 a 2030 é ainda mais preocupante, quase triplicando os valores a serem pagos, mantendo a prevalência de aumento da despesa nos grupos de idade mais velhos.

Os dados mostram que o resultado previdenciário total apurado a partir da razão demográfica projetada para o Cenário 1 apresentará déficit crescente ao longo dos anos (gráfico 6). 


\section{Gráfico 6}

Evolução do resultado previdenciário (Cenário 1) 2012-30

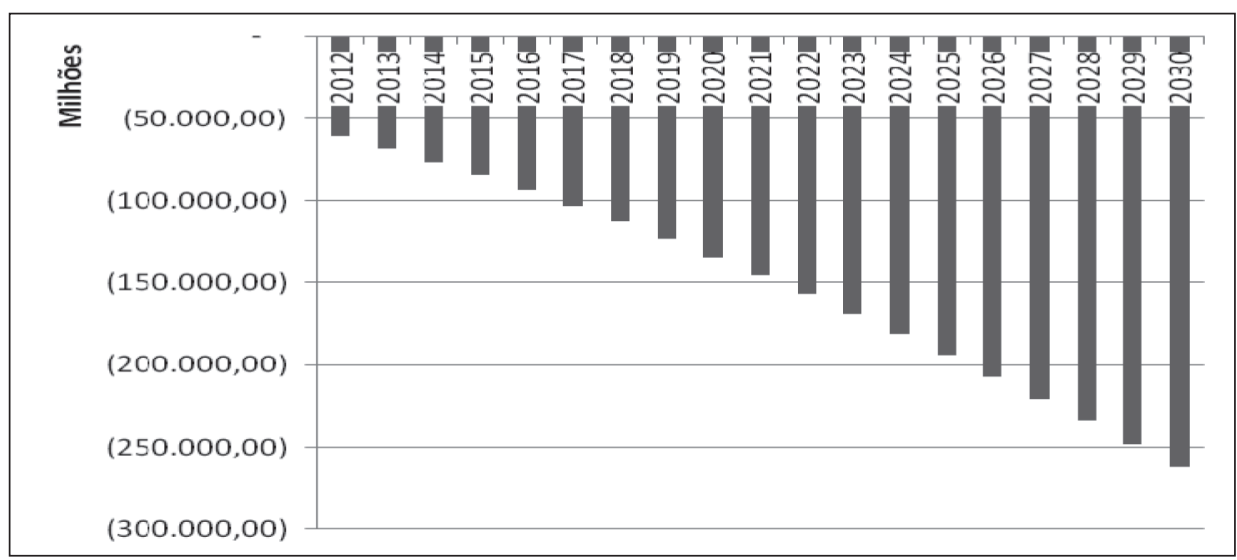

Fonte: Aeps Infologo.

A partir da análise do Cenário 1 é possível inferir que o envelhecimento populacional afetará de forma ainda mais acentuada a participação dos grupos de idade na arrecadação da receita previdenciária ao longo dos anos: a população jovem nascida depois do declínio da fecundidade, e, por isso, menor, terá de sustentar em um futuro próximo um grande número de população aposentada, nascida antes do declínio da fecundidade. Ou seja, considerando que no futuro os contribuintes atuais serão sustentados por uma massa de contribuintes muito menor, fica evidente o comprometimento do equilíbrio das contas previdenciárias.

Com relação à análise do segundo cenário projetado para o período de 2012 a 2030, os dados mostram que o déficit observado no primeiro cenário será ainda mais acentuado, revelando um cenário de completa instabilidade das contas previdenciárias (gráfico 7).

\section{Gráfico 7}

Evolução do resultado previdenciário (Cenário 2) 2012-30

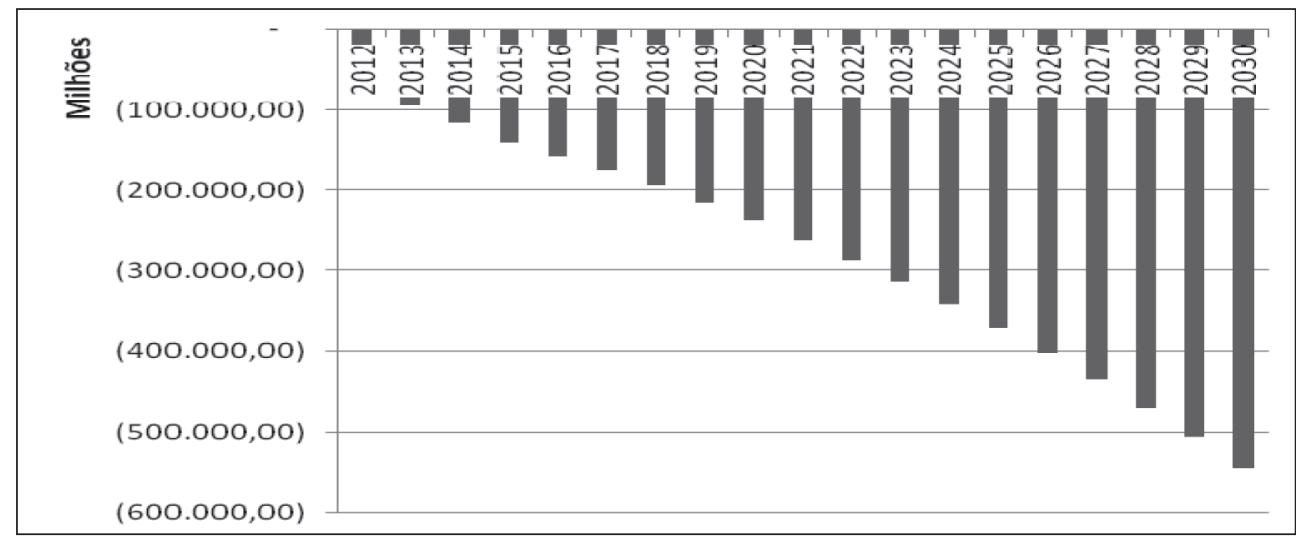

Fonte: Aeps Infologo. 


\subsection{Análise estocástica}

A partir da constatação de que a dinâmica demográfica afetou a sustentabilidade do regime geral de previdência social brasileiro no período de 2003 a 2030, foi efetuada análise estocástica por meio da simulação de Monte Carlo (MC), com os elementos envolvidos se comportando como variáveis aleatórias a partir de uma distribuição específica (uniforme) com o uso do software crystal ball, objetivando analisar as perspectivas de equilíbrio a partir dos resultados encontrados. Foram definidas quatro variáveis de entrada a partir do resultado apurado na análise determinística:

a) Número total de contribuintes pessoas físicas do RGPS em cada ano;

b) Valor anual médio de cada contribuição;

c) Número total de benefícios ativos, incluindo pensões, do RGPS em cada ano;

d) Valor anual médio de cada benefício.

Para a simulação da projeção do resultado previdenciário, inicialmente foi construído um modelo a partir dos resultados apurados na análise determinística, que resultou em uma distribuição de frequência uniforme de valores prováveis de serem apurados como resultado previdenciário em três períodos: 2003 a 2011, 2012 a 2030 e 2003 a 2030.

Na sequência, foi definida uma distribuição estocástica para descrever a situação de todos os valores prováveis (mínimos e máximos) dessas variáveis, gerando a primeira simulação do estudo. Em seguida, a partir da frequência de valores observada, foi efetuada a análise de sensibilidade para calcular as relações entre as quatro assumptions e a previsão (forecast), buscando mostrar essas relações em termos percentuais.

A partir da análise dos resultados, foi então efetuada a adição da aleatoriedade artificial (zero), utilizando-se novamente o software Crystal ball para determinar a probabilidade de o resultado previdenciário apresentar-se equilibrado.

Foram executados 10 mil ensaios (trials), com o uso da mesma sequência de números randômicos, e um intervalo de confiança de 95\%. Registra-se que uma característica presente no Crystal ball é a de que os valores extremos (outliers) estão incluídos nos cálculos mas não são apresentados nos gráficos.

No caso do fluxo do resultado previdenciário total para o período de 2003 a 2011, em que pese a manutenção do déficit ao longo dos anos, a análise estocástica mostra que havia pequena perspectiva de o resultado apresentar-se equilibrado em cada ano, sendo a melhor perspectiva apresentada no ano de 2011, que chegou a 4,34\% de possibilidade (gráfico 8).

Relativamente ao fluxo do resultado previdenciário total para o período de 2012 a 2030 a partir do Cenário 1, os dados do gráfico 9 mostram queda da perspectiva de equilíbrio: enquanto em 2012 havia 2,99\% de possibilidade de o resultado previdenciário apresentar-se equilibrado, em 2030 não haverá qualquer perspectiva nesse sentido. 
Gráfico 8

Probabilidade de equilíbrio no resultado previdenciário 2003-11
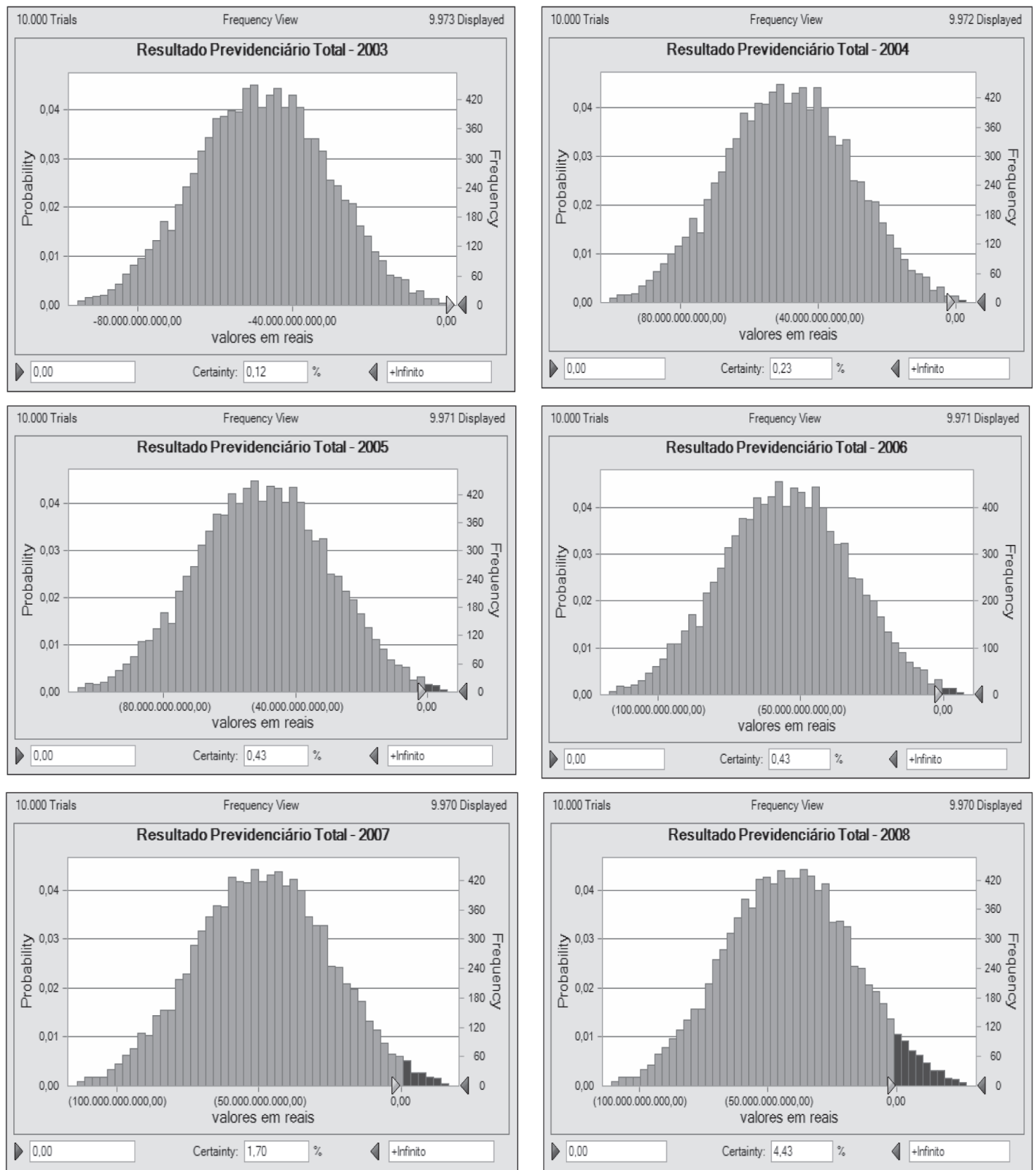

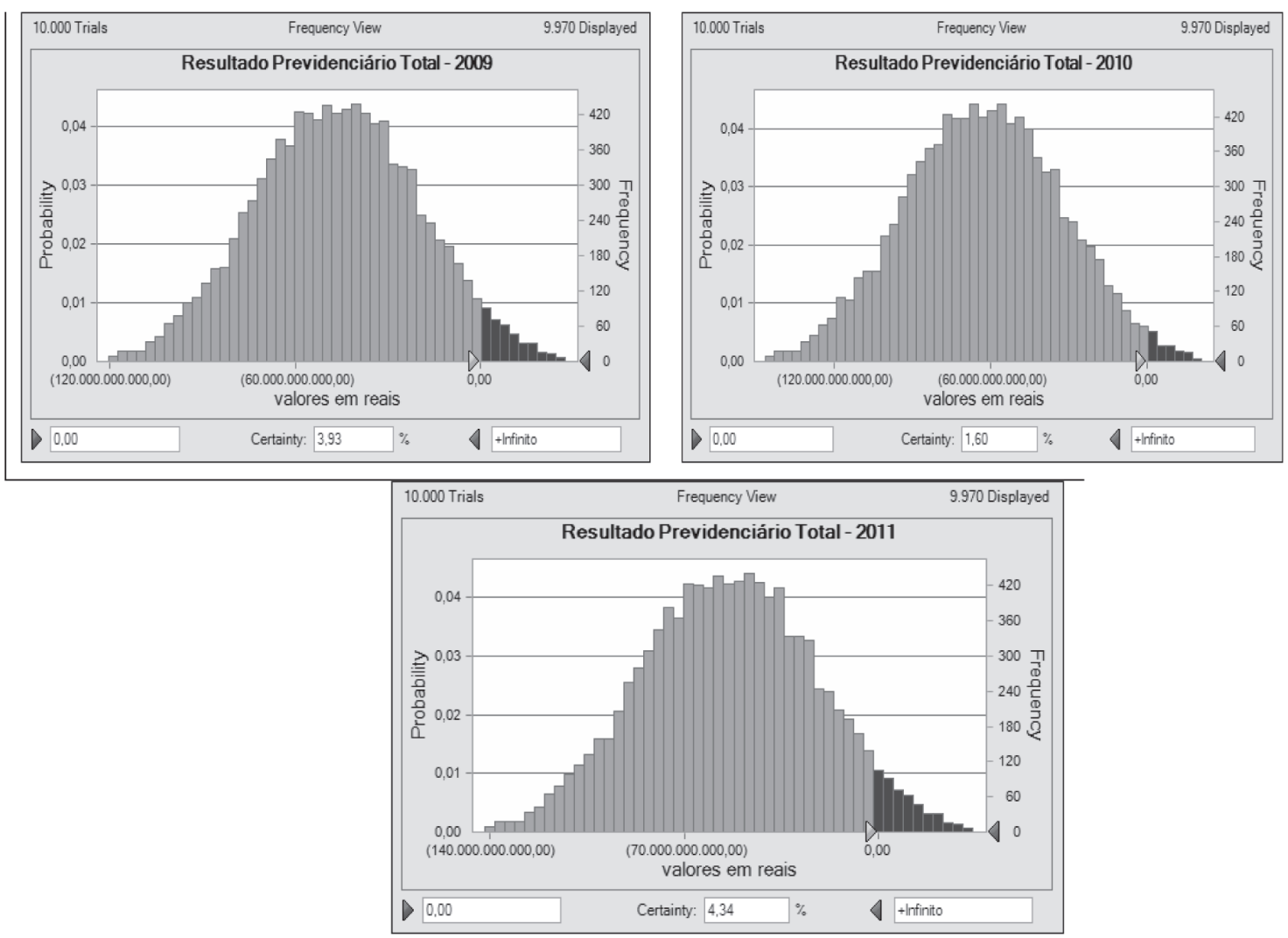

Fonte: Simulação do estudo realizada pelos autores.

Gráfico 9

Probabilidade de equilíbrio do resultado previdenciário 2012-30 (Cenário 1)
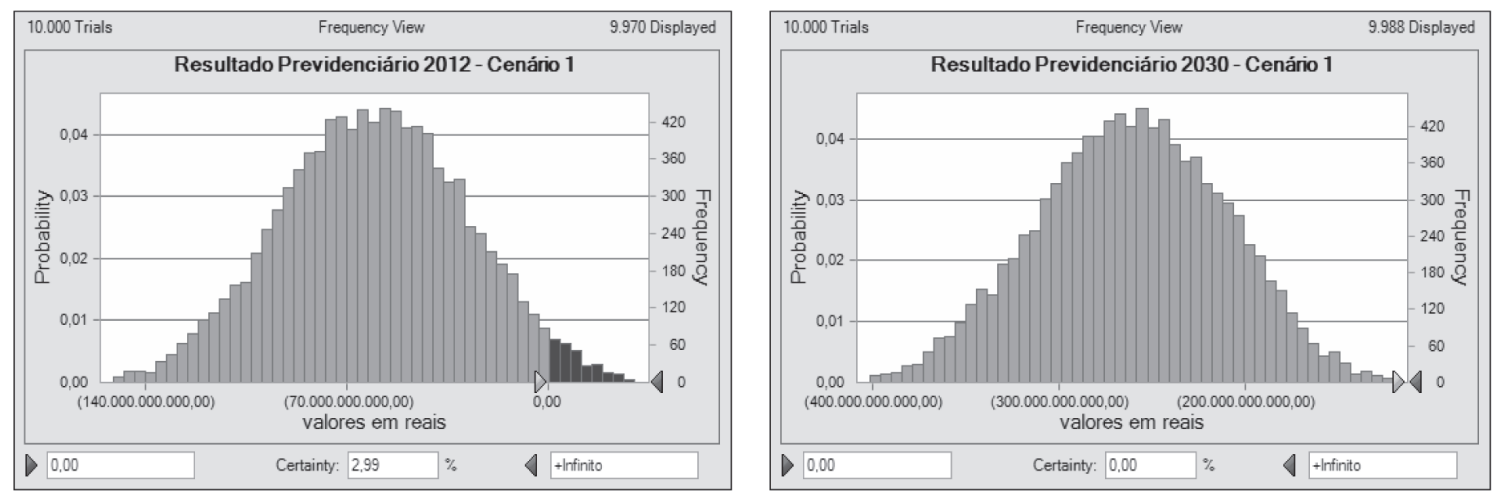

Fonte: Simulação do estudo realizada pelos autores. 
A mesma situação é observada na análise do fluxo do resultado previdenciário total para o período de 2012 a 2030 a partir do Cenário 2: no ano de 2012 a perspectiva de equilíbrio é ainda menor do que a observada no Cenário 1 , não havendo qualquer possibilidade de equilíbrio no ano de 2030 (gráfico 10).

\section{Gráfico 10}

Probabilidade de equilíbrio do resultado previdenciário 2012-30 (Cenário 2)
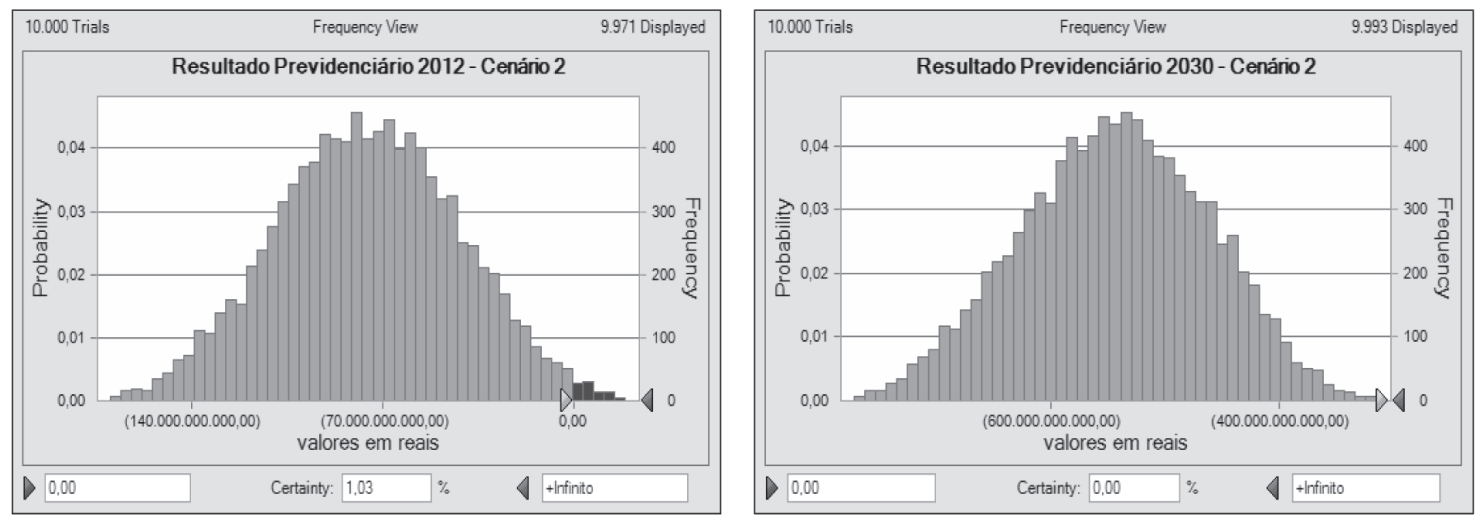

Fonte: Simulação do estudo realizada pelos autores.

Portanto, a partir dos resultados apurados tanto na análise determinística como na análise estocástica, há evidências de que as mudanças observadas na estrutura etária da população brasileira e na estrutura etária dos contribuintes e beneficiários do RGPS não só afetaram a evolução da receita previdenciária e da despesa previdenciária no período de 2003 a 2011, como afetarão de forma ainda mais acentuada no período de 2012 a 2030.

\section{Considerações finais}

O estudo teve como objetivo investigar os efeitos da dinâmica demográfica na sustentabilidade do regime geral de previdência social brasileiro e, mais especificamente, mensurar os efeitos da dinâmica demográfica nas receitas previdenciárias e nas despesas previdenciárias para o período de 2003 a 2030, e as perspectivas de equilíbrio a partir dos resultados encontrados.

A análise da receita previdenciária para o período de 2003 a 2011 apresentou trajetória de crescimento da arrecadação previdenciária em todos os anos analisados, dobrando, em termos comparativos, o total de valores arrecadados em 2011 em relação a 2003. Uma explicação para esses resultados pode ser a existência do fenômeno do bônus demográfico, que cria a possibilidade de maior crescimento econômico. Contudo, ao se analisar a participação de cada grupo de idade na arrecadação da receita entre os anos de 2003 a 2011, a partir da base de 
dados do MPS, verifica-se que houve queda da participação na faixa de 20 a 24 anos e de 35 a 49 anos de idade - justamente quando os indivíduos se encontram no ápice da idade ativa.

Com relação à análise da despesa previdenciária para o período de 2003 a 2011, os dados mostram que, embora seja observada a tendência de crescimento da despesa previdenciária em todos os anos analisados, quando se compara a participação de cada grupo de idade na evolução da despesa previdenciária em 2011 em relação a 2003 verifica-se que houve queda da participação da despesa nas faixas de 25 a 59 anos e aumento nos demais grupos de idade, bem como no caso das aposentadorias com pensões.

Quando é feita a análise do resultado previdenciário do período de 2003 a 2011, que se apresentou deficitário em todos os anos observados, há evidências, portanto, de que a dinâmica demográfica afetou o resultado previdenciário total tanto no conjunto da arrecadação das contribuições, com aumento do número de contribuintes, como no conjunto do pagamento dos benefícios do RGPS, com aumento do número de beneficiários.

As análises da receita previdenciária total para o período de 2012 a 2030 em ambos os cenários mostram a redução da capacidade contributiva das coortes mais jovens (até 29 anos) e menor ritmo de crescimento da receita, sendo ainda observada a prevalência de crescimento da arrecadação nos grupos de idade mais velhos. Portanto, o envelhecimento populacional afetará de forma ainda mais acentuada a participação dos grupos de idade na arrecadação da receita previdenciária ao longo dos anos: a população jovem nascida depois do declínio da fecundidade e, por isso, menor terá de sustentar em um futuro próximo um grande número de população aposentada, nascida antes do declínio da fecundidade.

Relativamente à análise da despesa previdenciária total para o período de 2012 a 2030, independentemente do cenário projetado no estudo, verifica-se trajetória de crescimento acentuado com o passar dos anos, sendo também observada modificação no perfil etário dos beneficiários, sinalizando que haverá crescimento do número de benefícios e, consequentemente, das despesas previdenciárias dos grupos de idade mais velhos.

Com isso, na análise do resultado previdenciário total para o período de 2003 a 2030 há evidências de que haverá crescimento significativo da necessidade de financiamento do RGPS ao longo dos anos, atingindo no ano de 2030 (Cenário 1) o valor de R\$ 261,9 bilhões de reais, cinco vezes maior do que a necessidade de financiamento observada no ano de 2003. A análise do Cenário 2 se mostra ainda mais preocupante, sinalizando o agravamento do déficit das contas previdenciárias.

Efetuando a análise estocástica do resultado previdenciário total para os períodos de 2003 a 2011, 2012 a 2030 e 2003 a 2030 verificou-se que, com o passar dos anos, a dinâmica demográfica (Cenário 1) comprometeu a capacidade de equilíbrio das contas do RGPS, uma vez que toda a distribuição observada em 2030 se apresentou no campo de valores negativos. Pela análise do Cenário 2, verificou-se que outros fatores também podem comprometer a capacidade de equilíbrio das contas previdenciárias. As variáveis "valor médio do benefício" e "número de beneficiários" foram identificadas como os principais fatores para os déficits apurados em todos os anos. 
Ao se analisar o grau de certeza de o resultado previdenciário alcançar equilíbrio a partir da suposição de que a receita previdenciária seja equivalente à despesa previdenciária em cada período (adição da aleatoriedade artificial), verificou-se que houve pequena evolução na perspectiva de equilíbrio do resultado previdenciário total no período de 2003 a 2011, que pode ser associada à recuperação da base de arrecadação do MPS. Na análise do período de 2012 a 2030, tanto para o Cenário 1 como para o Cenário 2 verificou-se que é remota a possibilidade de equilíbrio das contas previdenciárias.

Diante dos resultados encontrados, é possível inferir que a dinâmica demográfica não só afetou as contas previdenciárias no período de 2003 a 2011, como afetará de forma ainda mais significativa até o ano de 2030, sinalizando a premente necessidade de correções no modelo de financiamento atual do RGPS brasileiro.

\section{Referências}

BATISTA, Analia S. et al. Os idosos em situação de dependência e a proteção social no Brasil. Brasília: Ipea, abr. 2009. (Texto para discussão n. 1402).

BLOOM, David E. et al. Demographic change, social security systems, and savings. Journal of Monetary Economics, v. 54, p. 92-114, 2007.

BLUE, Laura; ESPENSHADE, Thomas J. Population momentum across the demographic transition. Population and Development Review, v. 37, n. 4, p. 721-747, 2011.

BRASIL. Constituição da República Federativa do Brasil: texto constitucional promulgado em 5 de outubro de 1988. Brasília: Senado Federal, Subsecretaria de Edições Técnicas, 2005.

BRITO, Fausto. Transição demográfica e desigualdades sociais no Brasil. R. Bras. Est. Pop., São Paulo, v. 25, n. 1, p. 5-26, jan./jun. 2008.

CROIX, David de la; PIERRARD, Olivier; SNEESSENS, Henri R. Aging and pensions in general equilibrium: labor market imperfections matter. Journal of Economic Dynamics and Control, v. 37, n. 1, p. 104-124, jan. 2013.

GONZALES-EIRAS, Martín G.; NIEPEL, Dirk. The future of social security. Journal of Monetary Economics, v. 55, p. 197-218, 2008.

IBGE. Instituto Brasileiro de Geografia e Estatística. Projeção da População do Brasil por sexo e idade para o período 1980-2050. Revisão 2008. Estudos e Pesquisas: informações demográficas e socieconômicas, n. 24. Rio de Janeiro: IBGE, 2008.

KIELING, Rejane I. Janela de oportunidade demográfica: um estudo sobre os impactos econômicos da transição demográfica no Brasil. Dissertação (mestrado em economia) — Departamento de Economia, Universidade Federal do Rio Grande do Sul, Porto Alegre, 2009.

MATIAS-PEREIRA, José. Administração pública comparada: uma avaliação das reformas administrativas do Brasil, EUA e União Europeia. Rev. Adm. Pública, v. 42, n. 1, p. 61-82, jan./fev. 2008. 
MATIAS-PEREIRA, José. Reforma da previdência em discussão: expectativas e possibilidades diante da janela de oportunidade demográfica. Observatório de la Economía Latinoamericana, n. 136, p. 1-36, 2010.

MATOS, Paulo R. F.; PINTO, Fabíola S. Análise da solvência do regime geral da previdência social no Brasil. XVII Prêmio Tesouro Nacional. Brasília, 2012.

MPS. Ministério da Previdência Social. Anuário estatístico do Ministério da Previdência Social. Empresa de Tecnologia e Informações da Previdência Social. Brasília: MPS/DATAPREV, 2012b. v. 20, p. $1-888$.

MPS. Ministério da Previdência Social. Projeção atuarial para o Regime Geral de Previdência Social - RGPS. Anexo IV - Metas fiscais. IV. 5 Projeções atuariais para o Regime Geral de Previdência

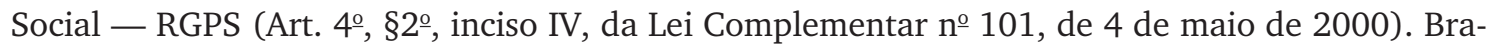
sília, 2012a.

NAM, Charles. Understanding population change. Itasca, IL: FE Peacock Publishers, 1994.

PAIVA, Paulo de T. A.; WAJNMAN, Simone. Das causas às consequências econômicas da transição demográfica no Brasil. Rev. Bras. Est. Pop., São Paulo, v. 22, n. 2, p. 303-322, jul./dez. 2005.

PESSOA, Izabel L. O envelhecimento na agenda da política social brasileira: avanços e limitações. Tese (doutorado em política social) — Programa de Pós-Graduação em Política Social, Departamento de Serviço Social, Universidade de Brasília, Brasília, 2009.

QUEIROZ, Bernardo L.; FÍGOLI, Moema G. B. The social protection system for the elderly in Brasil. In: Annual MEETING OF THE POPULATION ASSOCIATION OF AMERICA, 2011, Washington, DC. Anais...Washington: s.n., 2011.

RYDER, Norman B. Obituary: Frank Wallace Notestein (1902-1983), Population Studies, v. 38, n. 1, p. 5-20, 1984.

TCU. Tribunal de Contas da União. Minuta do relatório de fiscalização realizada no Ministério da Previdência Social, TC 015.529/2010-0.

WORLD BANK. Becoming old in an older Brazil: implications of population aging on growth, poverty, public finance and service delivery. Human Development Department Latin America and the Caribbean Region. Document of de World Bank, abr. 2011.

Diana Vaz de Lima é professora adjunta do Departamento de Ciências Contábeis e Atuariais da Universidade de Brasília (UnB). Doutora em ciências contábeis pelo Programa Multi-institucional e Inter-regional de Pós-Graduação em Ciências Contábeis UnB/UFPB/UFRN. E-mail: diana_lima@unb.br.

José Matias-Pereira é professor e pesquisador do Programa Multi-institucional e Inter-regional de PósGraduação em Ciências Contábeis UnB/UFPB/UFRN. Pós-doutorado em administração pela Faculdade de Economia, Administração e Contabilidade da Universidade de São Paulo (FEA/USP). Doutor em ciência política - área de governo e administração pública — pela Faculdade de Ciências Políticas e Sociologia da Universidade Complutense de Madri, Espanha. E-mail: matias@unb.br. 